\title{
Impact of cortical hemodynamic failure on both subsequent hemorrhagic stroke and effect of bypass surgery in hemorrhagic moyamoya disease: a supplementary analysis of the Japan Adult Moyamoya Trial
}

\author{
Jun C. Takahashi, MD, PhD, ${ }^{1}$ Takeshi Funaki, MD, PhD, ${ }^{2}$ Kiyohiro Houkin, MD, PhD, ${ }^{3}$ \\ Satoshi Kuroda, MD, PhD, ${ }^{4}$ Miki Fujimura, MD, $\mathrm{PhD},{ }^{5}$ Yasutake Tomata, $\mathrm{PhD},{ }^{6}$ and \\ Susumu Miyamoto, MD, PhD, ${ }^{2}$ on behalf of the JAM Trial Investigators
}

\begin{abstract}
1'Department of Neurosurgery, National Cerebral and Cardiovascular Center, Suita; ${ }^{2}$ Department of Neurosurgery, Kyoto University Graduate School of Medicine, Kyoto; ${ }^{3}$ Department of Neurological Cell Therapy, Hokkaido University Hospital, Sapporo; ${ }^{4}$ Department of Neurosurgery, Graduate School of Medicine and Pharmaceutical Sciences, University of Toyama; ${ }^{5}$ Department of Neurosurgery, Kohnan Hospital, Sendai; and ${ }^{6}$ Division of Epidemiology, Department of Health Informatics and Public Health, Tohoku University School of Public Health, Graduate School of Medicine, Sendai, Japan
\end{abstract}

\begin{abstract}
OBJECTIVE Here, the authors aimed to determine whether the presence of cerebral hemodynamic failure predicts subsequent bleeding attacks and how it correlates with the effect of direct bypass surgery in hemorrhagic moyamoya disease.
\end{abstract}

METHODS Data from the Japanese Adult Moyamoya (JAM) Trial were used in this study: 158 hemispheres in 79 patients. A newly formed expert panel evaluated the SPECT results submitted at trial enrollment and classified the cortical hemodynamic state of the middle cerebral artery territory of each hemisphere into one of the following three groups: SPECT stage (SS) 0 as normal, SS1 as decreased cerebrovascular reserve (CVR), and SS2 as decreased CVR with decreased baseline blood flow. In the nonsurgical cohort of the JAM Trial, the subsequent hemorrhage rate during the 5 -year follow-up was compared between the SSO (hemodynamic failure negative) and SS1+2 (hemodynamic failure positive) groups. The effect of direct or combined direct/indirect bypass surgery on hemorrhage prevention was examined in each subgroup.

RESULTS The hemodynamic grade was SS0 in 59 (37.3\%) hemispheres, SS1 in $87(55.1 \%)$, and SS2 in 12 (7.6\%). In the nonsurgical cohort, subsequent hemorrhage rates in the SS0 and SS1+2 groups were 12 cases per 1000 personyears and 67 cases per 1000 person-years, respectively. Kaplan-Meier analysis revealed that hemorrhagic events were significantly more common in the $S S 1+2$ group $(p=0.019$, log-rank test). Cox regression analysis showed that hemodynamic failure was an independent risk factor for subsequent hemorrhage (HR $5.37,95 \% \mathrm{Cl} 1.07-27.02)$. In the SS1+2 subgroup, bypass surgery significantly suppressed hemorrhagic events during 5 years $(p=0.001$, HR $0.16,95 \%$ $\mathrm{Cl} 0.04-0.57)$, with no significant effect in the SSO group ( $p=0.655, \mathrm{HR} 1.56,95 \% \mathrm{Cl} 0.22-11.10)$. Examination of effect modification revealed that the effect of surgery tended to differ nonsignificantly between these two subgroups $(p=$ 0.056).

CONCLUSIONS Hemodynamic failure is an independent risk factor for subsequent hemorrhage in hemorrhagic moyamoya disease. Direct bypass surgery showed a significant preventive effect in the hemodynamically impaired hemispheres. Thus, hemodynamic failure, as well as previously proposed factors such as choroidal anastomosis, should be considered for the surgical indication in hemorrhagic moyamoya disease.

Clinical trial registration no.: C000000166 (umin.ac.jp)

https://thejns.org/doi/abs/10.3171/2020.1.JNS192392

KEYWORDS hemorrhagic moyamoya disease; hemodynamic failure; periventricular anastomosis; subsequent bleeding; bypass surgery; vascular disorders

ABBREVIATIONS ACZ = acetazolamide; JAM = Japan Adult Moyamoya; $M C A=$ middle cerebral artery; $P C A=$ posterior cerebral artery; $r C B F=$ regional cerebral blood flow; SS = SPECT stage; VR = vascular reserve.

SUBMITTED September 2, 2019. ACCEPTED January 3, 2020.

INCLUDE WHEN CITING Published online March 13, 2020; DOI: 10.3171/2020.1.JNS192392. 
I NTRACRANIAL hemorrhage is the most critical adverse event in moyamoya disease, reportedly accompanied by a high rebleeding rate of $7 \%$ per year. ${ }^{10}$ The Japan Adult Moyamoya (JAM) Trial, a multicenter randomized controlled trial on hemorrhagic moyamoya disease, recently demonstrated that the posterior hemorrhage group (e.g., thalamus or posterior half of the lateral ventricle) has a high risk of rebleeding, that developed choroidal artery collateral vessels (choroidal anastomosis) strongly correlate with a high rebleeding rate, and that direct bypass surgery significantly prevents rebleeding, especially in the posterior hemorrhage group., 3,4,15 Hemodynamic failure is commonly detected in hemorrhagic moyamoya disease ${ }^{8}$ however, its correlation with the rebleeding rate or the preventive effect of bypass surgery is still unclear. In this study, we examined whether the presence of hemodynamic failure at the onset of hemorrhage predicts subsequent bleeding events and how it correlates with the effect of bypass surgery.

\section{Methods}

The JAM Trial was approved by the ethics committees of all the participating centers and was registered with the University Hospital Medical Information Network Clinical Trials Registry (clinical trial registration no.: C000000166, www.umin.ac.jp).

\section{Participants and Setting}

This ancillary cohort study used both the data set at the time of enrollment and the longitudinal data of the JAM Trial, which involved 80 participants recruited from 22 centers. The design of the JAM Trial has been described elsewhere. ${ }^{12}$ In brief, patients with moyamoya disease were eligible for the study if they had developed intracranial hemorrhage within 1 year before randomization, were 16-65 years old, were independent in daily life (modified Rankin Scale score 0-2), and had completed acute-phase treatment at least 1 month before randomization. Diagnosis of moyamoya disease was established according to the guidelines proposed in $1997 .{ }^{2}$ In addition to cerebral angiography, CT, and MRI, quantitative SPECT both at baseline (resting state) and after acetazolamide (ACZ) challenge 5 at least 1 month after hemorrhagic onset was required at the time of enrollment. Forty-two patients were randomly assigned to the surgical group (direct superficial temporal artery-middle cerebral artery [MCA] bypass with or without additional indirect procedures on both sides), and 38 patients were assigned to the nonsurgical group (only blood pressure control if required). Each patient underwent a 5-year scheduled follow-up.

An expert panel developed the current supplementary study design after the completion of patient follow-ups. One patient in the nonsurgical group was excluded because of an error in the quantitative SPECT study. Therefore, in 79 patients, hemisphere-based analyses were performed in 158 hemispheres: 84 hemispheres in the surgical group and 74 hemispheres in the nonsurgical group.

\section{Evaluation of Hemodynamic Failure}

An expert panel, assembled for the present analysis and completely blinded to other clinical information, evalu- ated the SPECT images that had been obtained at the time of enrollment into the JAM Trial and demonstrated regional cerebral blood flow $(\mathrm{rCBF})$ in the resting state and the ACZ challenge. Focus was placed on the cortex of the MCA territory at the level of the basal ganglia and lateral ventricles. The image of the bilateral cerebellum was also used as a reference. In each case, the expert panel was also referred to the quantitative values of $\mathrm{rCBF}$ that had been calculated using the ${ }^{123}$ I-labeled $N$-isopropyl$p$-iodoamphetamine (IMP) autoradiography method $^{7}$ or ${ }^{123}$ I-IMP microsphere method ${ }^{11}$ at each center. According to the following criteria, the hemodynamic state of each hemisphere was classified into one of the following three SPECT stages (SSs; Fig. 1): SS0, normal baseline rCBF with normal ACZ rCBF; SS1, normal baseline rCBF with reduced $\mathrm{ACZ}$ rCBF; and $\mathrm{SS} 2$, reduced baseline $\mathrm{rCBF}$ with reduced $\mathrm{ACZ} \mathrm{rCBF}$.

As required, the following quantitative criteria were used supplementarily per another trial of bypass surgery. ${ }^{9}$ Vascular reserve (VR; \%) was calculated using the following formula: [(ACZ rCBF - baseline $\mathrm{rCBF}) \times 100] /$ baseline rCBF. Moreover, SS0 probable was $\mathrm{VR} \geq 30 \%$; SS1 probable was $10 \% \leq \mathrm{VR}<30 \%$ or $\mathrm{VR}<10 \%$ with baseline $\mathrm{rCBF} \geq$ institutional normal value $\times 0.8$; and SS2 probable was $\mathrm{VR}<10 \%$ with baseline $\mathrm{rCBF}<$ institutional normal value $\times 0.8$.

An exclusively quantitative judgment policy using numerical rCBF values could not be adopted because deepseated brain injuries caused by initial intracerebral hemorrhage can reduce cortical $\mathrm{rCBF}$ values regardless of the presence of hemodynamic failure, well known as "cortical diaschisis." Another reason was the lack of standardization of the quantification method among the participating centers. The SPECT and MR images were simultaneously checked, and the potential cortical diaschisis was carefully estimated. When a SPECT image showed apparent crossed-cerebellar diaschisis (rCBF reduction in the contralateral cerebellum), ${ }^{14}$ decreased $\mathrm{rCBF}$ of the focused cortex was assumed to be a consequence of hypometabolism (cortical diaschisis induced by initial hemorrhagic lesions) rather than hemodynamic failure.

\section{Angiographic Evaluation of the Collateral Vessels and the Posterior Cerebral Artery}

Angiographic evaluation of the collateral vessels and posterior cerebral artery (PCA) has been described in detail in a previous supplementary study of the JAM Trial. ${ }^{3}$ Briefly, three types of collateral vessels in each hemisphere were assessed by an expert panel: lenticulostriate anastomosis, thalamic anastomosis, and choroidal anastomosis. Lenticulostriate anastomosis was judged to be positive when it dilated and extended beyond the level of the pericallosal artery. Thalamic anastomosis was judged to be positive in cases of extreme dilatation and extension beyond the position of the medial posterior choroidal artery. Choroidal anastomosis was positive in cases of extreme dilatation and extension with sudden deviation from the shape of the lateral ventricle to connect to the medullary artery. Another positive indicator was extension beyond the atrium of the lateral ventricle or connection of the medial posterior choroidal artery to the pericallosal artery by 

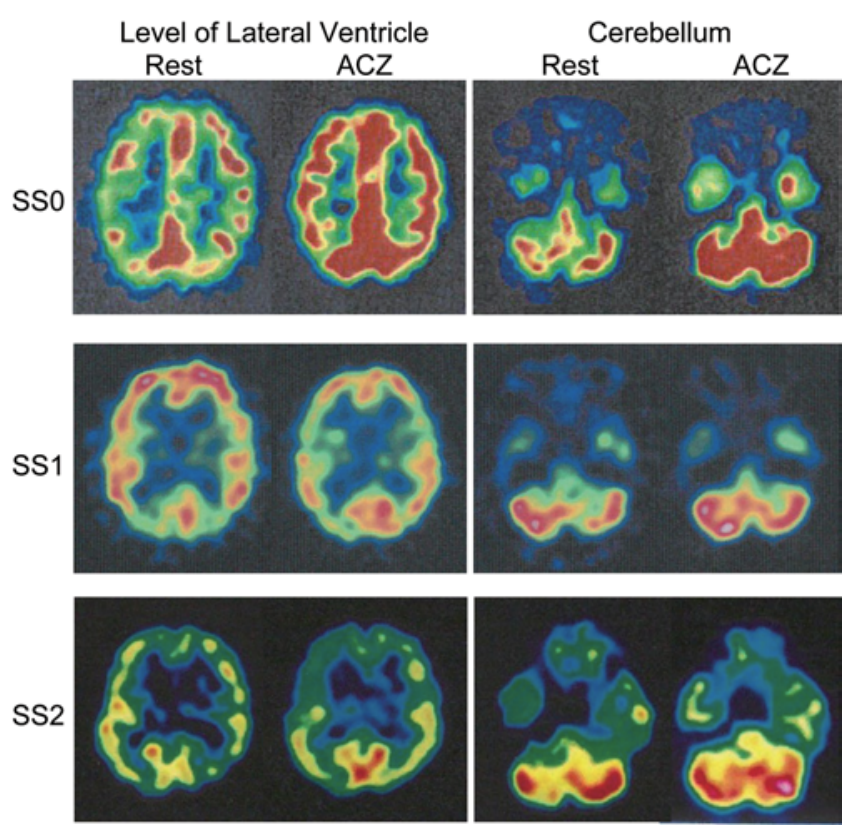

FIG. 1. The hemodynamic state was classified in each hemisphere. In the cases featured in this figure, bilateral hemispheres had the same grade. SS0, normal baseline rCBF with normal ACZ rCBF; SS1, normal baseline $\mathrm{rCBF}$ with reduced $\mathrm{ACZ}$ rCBF; and SS2, reduced baseline rCBF with reduced $A C Z$ rCBF.

penetration of the corpus callosum. PCA involvement was defined as the presence of stenotic changes $>50 \%$ in the $\mathrm{P}_{1}-\mathrm{P}_{3}$ segments. ${ }^{3}$

\section{Impact on Subsequent Hemorrhage and Surgical Effect}

In the nonsurgical group of the JAM Trial, the time to hemorrhagic events during the follow-up period was compared between the hemispheres with and without hemodynamic failure. Using the whole data set of the JAM Trial, the preventive effects of bypass surgery were evaluated separately in hemodynamically impaired and normal hemispheres. We also evaluated whether the effect of bypass surgery varied according to the hemodynamic severity (effect modification). The correlation between angiographic morphology (development of each collateral and
PCA involvement) and hemodynamic stage (0-2) was also assessed.

\section{Statistical Analyses}

The t-test, chi-square test, or Fisher's exact test was used for comparing baseline characteristics. The hazard ratio for subsequent bleeding according to the hemodynamic failure groups was calculated using a Cox proportional hazards regression model and adjusted for the following four potential confounders: patient age, sex, developed choroidal collateral, and PCA involvement. In order to examine whether the effect of bypass surgery on subsequent bleeding differed per hemodynamic failure, the hazard ratios of bypass surgery stratified by hemodynamic failure were also estimated with a Cox proportional hazards regression model. Effect modification of bypass surgery was tested using a Cox proportional hazards regression model incorporating the interaction term and was presented as a $\mathrm{p}$ value for interaction.

Two-sided p values $<0.05$ or $95 \%$ confidence intervals not including 1 were considered significant. All analyses were performed with IBM SPSS software, version 24 (IBM Corp.).

\section{Results}

Among all 158 hemispheres, the hemodynamic grade of the MCA territory was SS0 in 59 (37.3\%) hemispheres, SS1 in 87 (55.1\%), and SS2 in 12 (7.6\%).

\section{Hemodynamic Failure and Subsequent Bleeding Attacks in the Nonsurgical Group}

Analyses of hemodynamic failure and subsequent bleeding attacks involved inspection of the angiographic findings, and one patient in the nonsurgical group was excluded because the original angiography submitted at enrollment was unavailable. Baseline variables for the nonsurgical group are listed in Table 1. Among the 72 nonsurgical hemispheres, $34(47.2 \%)$ were assigned to the SS0 (hemodynamic failure negative) group and 38 (52.8\%) were assigned to either the SS1 or SS2 (SS1+2, hemodynamic failure positive) group.

Two hemispheres (5.9\%) in the SS0 group and $9(23.7 \%)$

TABLE 1. Baseline variables of the 72 hemispheres in the nonsurgical group

\begin{tabular}{lccc}
\hline \multicolumn{1}{c}{ Variable } & $\begin{array}{c}\text { Hemodynamic Failure } \\
\text { Negative, SS0 }\end{array}$ & $\begin{array}{c}\text { Hemodynamic Failure } \\
\text { Positive, SS1+2 }\end{array}$ & p Value \\
\hline No. of hemispheres (\%) & $34(47.2)$ & $38(52.8)^{*}$ & \\
\hline Mean age in yrs & $40.3 \pm 11.2$ & $41.2 \pm 12.7$ & 0.735 \\
\hline Female, no. (\%) & $24(70.6)$ & $28(73.7)$ & 0.770 \\
\hline Hypertension, no. (\%) & $4(11.8)$ & $8(21.1)$ & 0.291 \\
\hline Diabetes mellitus, no. (\%) & $1(2.9)$ & $3(7.9)$ & 0.617 \\
\hline Hyperlipidemia, no. (\%) & $2(5.9)$ & $2(5.3)$ & 1.000 \\
\hline Lenticulostriate anastomosis, no. (\%) & $4(11.8)$ & $9(23.7)$ & 0.189 \\
\hline Thalamic anastomosis, no. (\%) & $10(29.4)$ & $12(31.6)$ & 0.842 \\
\hline Choroidal anastomosis, no. (\%) & $14(41.2)$ & $17(44.7)$ & 0.761 \\
\hline PCA involvement, no. (\%) & $3(8.8)$ & $14(36.8)$ & 0.005 \\
\hline * SS1 = 33 (45.8\%); SS2 = 5 (6.9\%). & & &
\end{tabular}




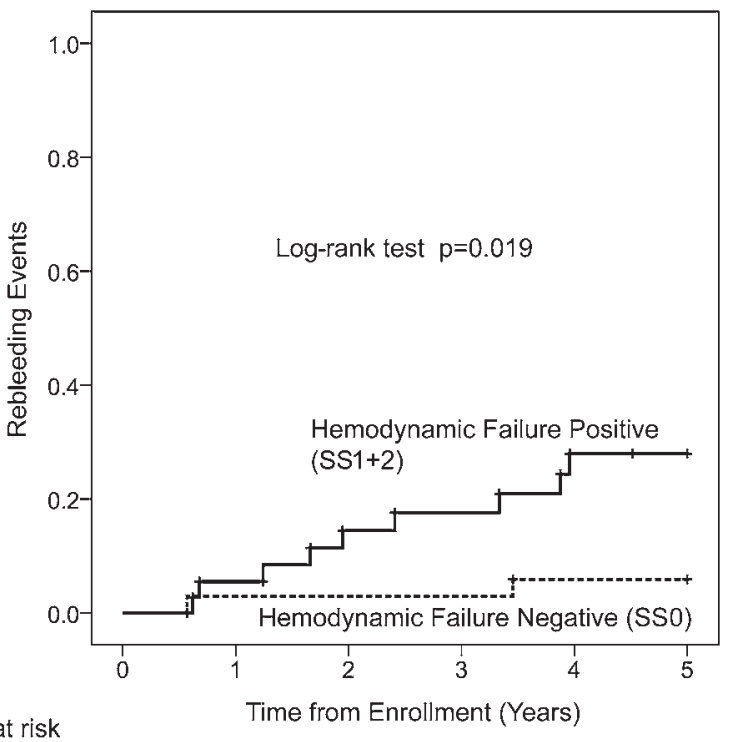

Number at risk

$\begin{array}{rrrrrrr}\text { SS1+2 } & 38 & 33 & 27 & 25 & 19 & 17 \\ \text { SSO } & 34 & 33 & 33 & 33 & 31 & 31\end{array}$

FIG. 2. Kaplan-Meier survival curve for subsequent hemorrhage in the nonsurgical hemispheres.

in the SS1+2 group developed subsequent hemorrhage during the 5-year follow-up period. The bleeding rates for the SS0 and SS1+2 groups were 12 cases per 1000 personyears and 67 cases per 1000 person-years, respectively. Kaplan-Meier analysis revealed that hemorrhagic events were significantly more common in the SS1+2 group ( $\mathrm{p}$ $=0.019$, log-rank test; Fig. 2). Cox regression analysis adjusted for four potential confounders revealed that positive hemodynamic failure was an independent risk factor for subsequent hemorrhage (HR 5.37, 95\% CI 1.07-27.02; Table 2).

\section{Hemodynamic Failure and Preventive Effect of Bypass Surgery}

Figure 3 demonstrates the Kaplan-Meier survival curves focused on subsequent hemorrhage in surgical and nonsurgical hemispheres. In the 59 SSO hemispheres, no significant difference was observed between the surgical and nonsurgical groups $(\mathrm{p}=0.655$, log-rank test; HR 1.56, 95\% CI 0.22-11.10; Fig. 3A). In contrast, in the $99 \mathrm{SS} 1+2$ hemispheres (positive hemodynamic failure), bypass surgery significantly prevented hemorrhagic events during the 5-year follow-up period ( $\mathrm{p}=0.001, \log$-rank test; HR $0.16,95 \%$ CI 0.04-0.57; Fig. 3B). When adjusted for four potential confounders, the result was almost the same (HR $0.15,95 \%$ CI $0.04-0.57)$. The examination of effect modification revealed that the effect of bypass surgery tended to differ between the hemodynamically impaired hemispheres and normal hemispheres, although the difference was not statistically significant $(\mathrm{p}=0.056)$.

\section{Vascular Morphology and Hemodynamic Failure}

Among the 156 hemispheres that were angiographically evaluated, lenticulostriate, thalamic, and choroidal anastomoses were judged to be positive in 37 (23.7\%), 49 (31.4\%),
TABLE 2. Multiple-adjusted hazard ratios for subsequent hemorrhage in the 72 hemispheres of the nonsurgical group

\begin{tabular}{llcllc}
\hline \multirow{2}{*}{ Analysis } & \multicolumn{2}{c}{ Crude } & & \multicolumn{2}{c}{ Multivariate Adjustment* } \\
\cline { 2 - 3 } & $\mathrm{HR}$ & $95 \% \mathrm{Cl}$ & & $\mathrm{HR}$ & $95 \% \mathrm{Cl}$ \\
\hline Hemodynamic status & & & & \\
\hline SS0 & 1.00 & Reference & 1.00 & Reference \\
\hline SS1+2 & 5.19 & $1.12-24.12$ & 5.37 & $1.07-27.02$ \\
\hline Choroidal anastomosis & & & & \\
\hline Negative & 1.00 & Reference & 1.00 & Reference \\
\hline Positive & 4.21 & $1.12-15.92$ & 6.26 & $1.43-27.39$ \\
\hline
\end{tabular}

${ }^{*}$ Cox proportional hazards model including the following five factors: hemodynamic status (SS1+2/SS0), choroidal anastomosis (positive/negative), age (continuous variable), sex (female/male), and PCA involvement (positive/ negative).

and $65(41.7 \%)$ hemispheres, respectively. PCA involvement was confirmed in $42(26.9 \%)$ hemispheres. Figure 4 demonstrates the hemodynamic stages in each collateral group. Development of lenticulostriate, thalamic, and choroidal collaterals showed no relation to hemodynamic stage, whereas PCA involvement led to a significant tendency of hemodynamic deterioration ( $\mathrm{p}$ for trend $=0.001$ ).

\section{Discussion}

The present study proposes two important findings. First, the existence of hemodynamic failure in hemorrhagic moyamoya disease is significantly correlated with subsequent hemorrhagic events in the nonsurgical (natural course) group. To the best of our knowledge, there has been only one report that focuses on hemodynamic failure and subsequent hemorrhagic stroke in hemorrhagic moyamoya disease. Jo et al. reported that decreased baseline rCBF on SPECT seems to be associated with recurrent bleeding. ${ }^{8}$ Their study cohort, however, was a mixture of both surgically treated and nonsurgical patients. The present study clearly elucidated significant findings in the natural course of hemorrhagic moyamoya disease. Second, direct bypass surgery significantly reduced the bleeding rate in hemodynamically impaired hemispheres. No previous reports have focused on hemodynamic failure and the effect of bypass surgery with respect to hemorrhage prevention.

Thus far, it is unclear why hemodynamic failure increases the rate of subsequent bleeding attacks in hemorrhagic moyamoya disease. A recent analysis of a nonsurgical cohort of the JAM Trial revealed that rebleeding was significantly frequent in cases with the development of positive choroidal periventricular anastomosis. ${ }^{4}$ In the present study, hemodynamic failure and choroidal anastomosis were independent risk factors for subsequent bleeding (Table 2). However, it is noteworthy that no direct correlation was observed between choroidal anastomosis and hemodynamic grades. PCA involvement was the only factor with a positive correlation with impaired hemodynamics. PCA occlusion can cause hemodynamic failure either by hypoperfusion of the posterior cerebral territory or by decreased collateral supply to the anterior circulation..$^{13}$ It is possible that hypoperfusion caused by PCA involvement induces choroidal collaterals that gradually 
A Hemodynamic Failure Negative (SSO, $n=59$ )

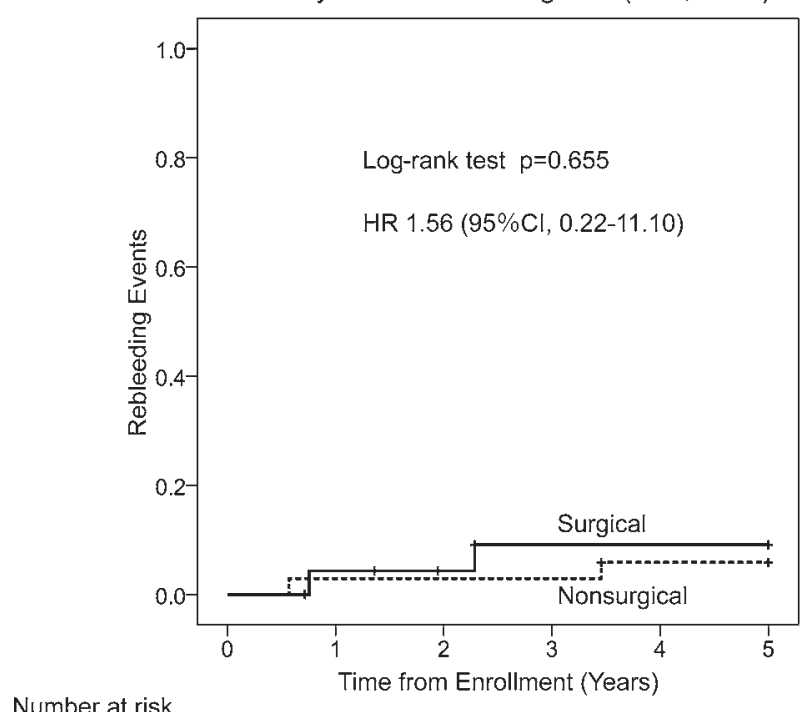

Number at risk

\begin{tabular}{|c|c|c|c|}
\hline Surgical & 25 & 22 & 20 \\
\hline lonsurgical & 34 & 33 & 33 \\
\hline
\end{tabular}

B Hemodynamic Failure Positive (SS1+2, n=99)

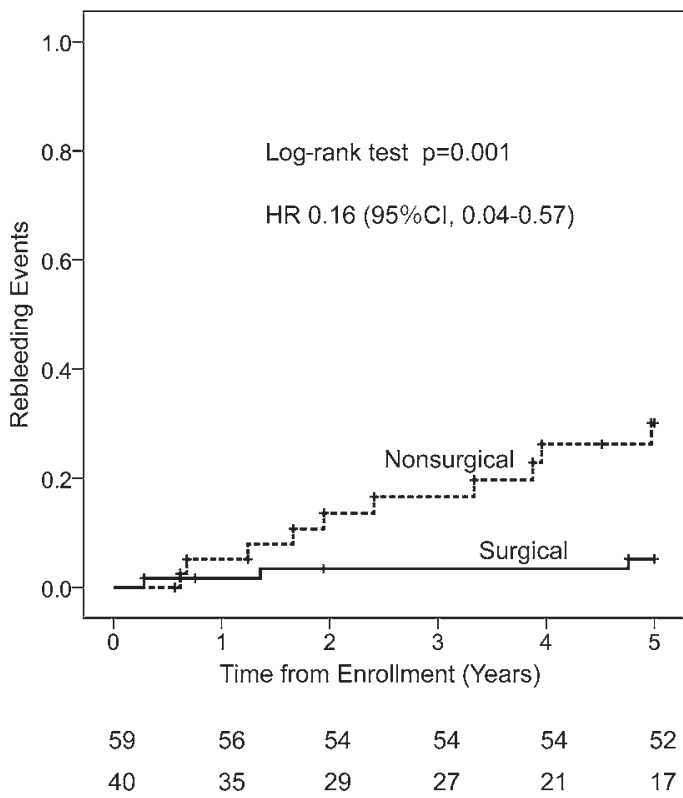

FIG. 3. Kaplan-Meier survival curves for subsequent hemorrhage in surgical and nonsurgical hemispheres. A: In 59 SSO hemispheres. B: In 99 SS1+2 hemispheres.

improve hemodynamic state; thus, choroidal anastomosis does not correlate directly with hemodynamic failure. In fact, choroidal anastomosis tended to be prominent with PCA involvement in the nonsurgical cohort of the JAM Trial, although the relationship between the two was not statistically significant. ${ }^{4}$ The reason for frequent bleeding in cases of hemodynamic failure warrants further investigation.

Bypass surgery showed significant potential in the prevention of subsequent bleeding in the hemispheres with impaired hemodynamics, while no significant effect was observed in those with normal hemodynamics. The JAM Trial revealed that direct bypass surgery prevents rebleeding attacks, especially in the posterior hemorrhage group. ${ }^{12,15}$ The present results may support the hypothesis that decreased cortical perfusion pressure causes a great demand for blood, leading to abundant postoperative bypass flow and significant reduction in the hemodynamic burden on the fragile collateral vessels. Further quantitative analyses of preoperative hemodynamic impairment and postoperative angiographic evaluation of the bypass flow may help to assess this hypothesis.

This study has certain limitations. First, it was not a prespecified cohort study; therefore, all the potential confounders could not be considered. Second, the hemodynamic evaluation was not quantitative. A purely quantitative judgment policy could not be adopted because brain damage caused by initial hemorrhage can lower the cortical $\mathrm{rCBF}$ values, regardless of the presence or absence of hemodynamic failure (cortical diaschisis). ${ }^{1}$ This made
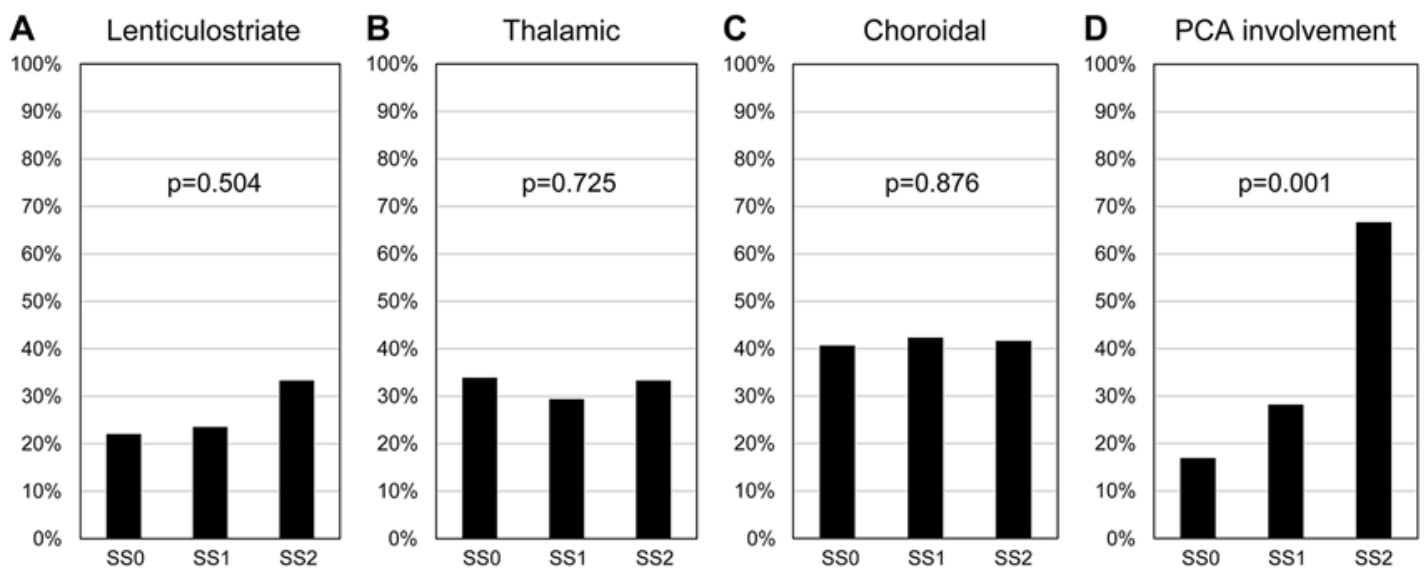

FIG. 4. Vascular morphology and hemodynamic failure. A: Positive lenticulostriate anastomosis. B: Positive thalamic anastomosis. C: Positive choroidal anastomosis. D: PCA involvement. The y-axes represent the percentage of a positive vascular finding. 
independent analyses of SS1 and SS2 rather difficult, leading to adoption of the combined group (SS1+2). As mentioned in detail in Methods, however, potential cortical diaschisis was carefully estimated based on evaluation of the SPECT images. The hemodynamic stage determined using this method showed a significant correlation with PCA occlusion (Fig. 4). Considering that PCA occlusion is an established factor of impaired hemodynamics in moyamoya disease, ${ }^{6,13,16}$ the use of the present semiquantitative evaluation could be warranted.

In sum, we emphasize that the present findings can provide some useful information for clinical decisionmaking. The JAM Trial and its subsequent analyses have already proposed that a patient with posterior hemorrhage and/or prominent choroidal collateral vessels may be a good candidate for bypass surgery for the prevention of fatal rebleeding attacks., ${ }^{3,4,12,15}$ Per the present findings, it appears reasonable to consider hemodynamic failure, as well as the initial bleeding site and choroidal collateral development, when making a decision about the surgical indication. Further prospective studies are needed to confirm this concept, and such studies could provide a more sophisticated management strategy for patients with hemorrhagic moyamoya disease.

\section{Conclusions}

The presence of hemodynamic failure is an independent risk factor for subsequent hemorrhagic stroke in hemorrhagic moyamoya disease. The preventive effect of bypass surgery was significant in hemodynamically impaired hemispheres. These findings suggest that hemodynamic failure, as well as previously proposed factors such as choroidal anastomosis, should be considered for the surgical indication in hemorrhagic moyamoya disease.

\section{Acknowledgments}

The Japanese Adult Moyamoya Trial has been funded since 1999 by a grant from the Japanese Ministry of Health, Labour and Welfare as a major project of the Research Committee on Spontaneous Occlusion of the Circle of Willis (moyamoya disease).

\section{References}

1. Baron JC, Levasseur M, Mazoyer B, Legault-Demare F, Mauguière F, Pappata $\mathrm{S}$, et al: Thalamocortical diaschisis: positron emission tomography in humans. J Neurol Neurosurg Psychiatry 55:935-942, 1992

2. Fukui M: Guidelines for the diagnosis and treatment of spontaneous occlusion of the circle of Willis ('moyamoya' disease). Research Committee on Spontaneous Occlusion of the Circle of Willis (Moyamoya Disease) of the Ministry of Health and Welfare, Japan. Clin Neurol Neurosurg 99 (2 Suppl 2):S238-S240, 1997

3. Funaki T, Takahashi JC, Houkin K, Kuroda S, Takeuchi S, Fujimura M, et al: Angiographic features of hemorrhagic moyamoya disease with high recurrence risk: a supplementary analysis of the Japan Adult Moyamoya Trial. J Neurosurg 128:777-784, 2018

4. Funaki T, Takahashi JC, Houkin K, Kuroda S, Takeuchi S, Fujimura M, et al: High rebleeding risk associated with choroidal collateral vessels in hemorrhagic moyamoya disease: analysis of a nonsurgical cohort in the Japan Adult Moyamoya Trial. J Neurosurg 130:525-530, 2019
5. Hirano T, Minematsu K, Hasegawa Y, Tanaka Y, Hayashida $\mathrm{K}$, Yamaguchi T: Acetazolamide reactivity on 123I-IMP single photon emission computed tomography in patients with major cerebral artery occlusive disease: correlation with positron emission tomography parameters. J Cereb Blood Flow Metab 14:763-770, 1994

6. Hishikawa T, Tokunaga K, Sugiu K, Date I: Assessment of the difference in posterior circulation involvement between pediatric and adult patients with moyamoya disease. J Neurosurg 119:961-965, 2013

7. Iida $H$, Itoh $H$, Nakazawa M, Hatazawa J, Nishimura $H$, Onishi Y, et al: Quantitative mapping of regional cerebral blood flow using iodine-123-IMP and SPECT. J Nucl Med 35:2019-2030, 1994

8. Jo KI, Kim MS, Yeon JY, Kim JS, Hong SC: Recurrent bleeding in hemorrhagic moyamoya disease: prognostic implications of the perfusion status. J Korean Neurosurg Soc 59:117-121, 2016

9. Kataoka H, Miyamoto S, Ogasawara K, Iihara K, Takahashi JC, Nakagawara J, et al: Results of prospective cohort study on symptomatic cerebrovascular occlusive disease showing mild hemodynamic compromise [Japanese ExtracranialIntracranial Bypass Trial (JET)-2 Study]. Neurol Med Chir (Tokyo) 55:460-468, 2015

10. Kobayashi E, Saeki N, Oishi H, Hirai S, Yamaura A: Longterm natural history of hemorrhagic moyamoya disease in 42 patients. J Neurosurg 93:976-980, 2000

11. Kuhl DE, Barrio JR, Huang SC, Selin C, Ackermann RF, Lear JL, et al: Quantifying local cerebral blood flow by Nisopropyl-p-[123I]iodoamphetamine (IMP) tomography. J Nucl Med 23:196-203, 1982

12. Miyamoto S, Yoshimoto T, Hashimoto N, Okada Y, Tsuji I, Tominaga T, et al: Effects of extracranial-intracranial bypass for patients with hemorrhagic moyamoya disease: results of the Japan Adult Moyamoya Trial. Stroke 45: 1415-1421, 2014

13. Mugikura S, Takahashi S, Higano S, Shirane R, Kurihara N, Furuta S, et al: The relationship between cerebral infarction and angiographic characteristics in childhood moyamoya disease. AJNR Am J Neuroradiol 20:336-343, 1999

14. Pantano P, Baron JC, Samson Y, Bousser MG, Derouesne C, Comar D: Crossed cerebellar diaschisis. Further studies. Brain 109:677-694, 1986

15. Takahashi JC, Funaki T, Houkin K, Inoue T, Ogasawara K, Nakagawara J, et al: Significance of the hemorrhagic site for recurrent bleeding: prespecified analysis in the Japan Adult Moyamoya Trial. Stroke 47:37-43, 2016

16. Yamada I, Himeno Y, Suzuki S, Matsushima Y: Posterior circulation in moyamoya disease: angiographic study. Radiology 197:239-246, 1995

\section{Disclosures}

The authors report no conflict of interest concerning the materials or methods used in this study or the findings specified in this paper.

\section{Author Contributions}

Conception and design: Miyamoto, Takahashi, Funaki, Houkin. Acquisition of data: Miyamoto, Takahashi, Funaki, Kuroda, Fujimura. Analysis and interpretation of data: all authors. Drafting the article: Takahashi. Critically revising the article: all authors. Reviewed submitted version of manuscript: all authors. Approved the final version of the manuscript on behalf of all authors: Miyamoto. Statistical analysis: Tomata.

\section{Correspondence}

Susumu Miyamoto: Graduate School of Medicine, Kyoto University, Kyoto, Japan. miy@kuhp.kyoto-u.ac.jp. 